\title{
ON THE RIGIDITY OF GRADED ALGEBRAS ${ }^{1}$
}

\author{
JANE PURCELL COFFEE
}

\begin{abstract}
If $\mathcal{G}$ is a graded algebra (separated and complete) over a field of characteristic zero and $\mathcal{G}$ is rigid in the category of algebras, then $\mathcal{G}$ is rigid in the category of filtered algebras.
\end{abstract}

1. Introduction. If $\mathcal{G}$ is a graded algebra (separated and complete) over a field $\mathcal{K}$ of characteristic zero and $\mathcal{G}$ is rigid in the category of algebras, then, as we show here, $\mathcal{G}$ is rigid in the category of filtered algebras and hence isomorphic, as a filtered algebra, to any $\mathscr{F}$ whose (complete) associated graded algebra is $\mathcal{G}$. Thus if a graded algebra is rigid in its operational structure, then it must also be rigid in its filtration structure.

The restriction that the graded algebra under consideration be an algebra over a field of characteristic zero (or more precisely, an algebra over a commutative unital coefficient ring $\mathscr{K}$ which is itself an algebra over $Q$ ) is required for the proof of Theorem 2 which we give here. The result that in a trivial deformation the infinitesimal of the deformation is a coboundary is critical to the proof and the following examples due to $M$. Gerstenhaber ([2]-[5]) show that this result need not hold for algebras defined over a field of characteristic $p>0$.

Let $\mathcal{K}$ be a field of characteristic 2 and the algebra $A=\mathscr{K}[x] / x^{2}$. Consider the deformation $A_{t}=\mathscr{K}[x][[t]] /\left(x^{2}+t\right)$. The multiplication on $A_{t}$ is $f_{t}=\pi+t F$ where $F(1,1)=F(1, x)=F(x, 1)=0, F(x, x)=1$ and $\pi$ is the original multiplication on $A . A_{t}$ is a nontrivial deformation of $A$ since there is no power series of the form $w=x+a_{1} t+a_{2} t^{2}+\ldots$ with coefficients $a_{1}, a_{2}, \ldots$ in $A$ such that with the multiplication in $A, w^{2} \equiv t$ $\bmod t^{2}$. Thus $F$ is not a coboundary since if it were, $A_{t}$ would be a trivial deformation. Now consider another deformation of $A, B_{t}=\mathcal{K}[x][[t]] /\left(x^{2}+\right.$ $t^{2}$ ). The multiplication on $B_{t}$ is defined by $g_{t}=\pi+t F$ where $F$ is the same as defined above. $B_{t}$ is a trivial deformation of $A$ under the mapping $\Phi_{t}=x+t$ which is an automorphism since $\mathcal{K}$ is a field of characteristic 2 . Thus $B_{t}$ is a trivial deformation but the infinitesimal of this deformation, $F$, is not a coboundary.

Given the history of results in algebraic deformation theory it is unlikely

Received by the editors January 26, 1978.

AMS (MOS) subject classifications (1970). Primary 18H15; Secondary 16A58.

Key words and phrases. Filtered algebras, associated graded algebras, algebraic deformations.

'This research was supported in part by a Naval Academy Research Grant. 
that the major result of this paper translates exactly to algebras over fields of characteristic $\boldsymbol{p}>0$.

The algebra $\mathcal{G}$ under consideration need not be associative. It is sufficient that $\mathcal{G}$ be an element of a category in which the $\mathcal{K}$-split extensions of $\mathcal{G}$ by $\mathcal{G}$ considered as a $\mathcal{G}$-bimodule form a group $H^{2}(\mathcal{G} \mathcal{G})$. The list of such categories includes associative, Lie, commutative associative, and nilpotent associative.

2. Gerstenhaber [2] showed that given a complete, separated filtered ring $\mathscr{F}$ there exists a one parameter family of deformations $f_{t}=\pi+t F_{1}+t^{2} F_{2}+$ $t^{3} F_{3}+\ldots$ of the (complete) associated graded ring $\mathcal{G}$ and $\mathcal{G}$ with multiplication $f_{1}$ is isomorphic to $\mathscr{F}$ as a filtered ring. Thus $\mathcal{F}$ is a deformation of $\mathcal{G}$ under $f_{t}$ and the infinitesimal of this deformation is $F_{1}$.

There is an alternate way of viewing $\mathcal{F}$ as a deformation of its associated graded ring $\mathcal{G}$. Following Gerstenhaber [3], we consider

$$
\text { App } \mathscr{F}=\left\{\sum_{i=-n}^{\infty} a_{i} t^{i} \mid a_{i} \in F^{i} \mathscr{F}\right\} \subseteq \mathscr{F}((t))
$$

Let

$$
F^{n} \mathrm{App} \mathcal{F}=\left\{\sum_{i=-n}^{\infty} a_{i} t^{i} \mid a_{i} \in F^{i+n} \mathcal{F}\right\}
$$

and let $\mathrm{App}_{n} \mathscr{F}=\mathrm{App} \mathscr{F} / F^{n+1} \mathrm{App} \mathscr{F} ; \mathrm{App}_{0} \mathscr{F}=\mathcal{G}$. There is a natural inclusion from $F^{n+1} \mathscr{F} / F^{n+2} \mathscr{F} \rightarrow F^{n} \mathscr{F} / F^{n+2} \mathscr{F}$. The direct sum over all $n$ of these maps yields:

$$
0 \rightarrow \bigoplus F^{n+1} \mathscr{F} / F^{n+2} \mathscr{F} \rightarrow \mathrm{App}_{1} \mathscr{F}
$$

where $\bigoplus F^{n+1} \mathscr{F} / F^{n+2} \mathscr{F}$ is $\mathcal{G}$ with the degree shifted by one and zero mulitplication. Denote this subobject of $\mathrm{App}_{1} \mathscr{F}$ by $\mathrm{sh} \mathcal{G}$. Thus there exists an exact sequence: $0 \rightarrow \operatorname{sh} \mathcal{G} \rightarrow \mathrm{App}_{1} \mathscr{F} \rightarrow \mathcal{G} \rightarrow 0$ which is a singular extension and therefore an element of $\operatorname{Baer}^{2}(\mathcal{G}, \operatorname{sh} \mathcal{G})$.

THEOREM 1. The singular extension: $0 \rightarrow \operatorname{sh} \mathcal{G} \rightarrow \mathrm{App}_{1} \mathcal{F} \rightarrow \mathcal{G} \rightarrow 0$ corresponds with the classical infinitesimal of the deformation from $\mathcal{G}$ to $\mathscr{F}$.

Proof. Let $f_{t}=\pi+t F_{1}+t^{2} F_{2}+\ldots$ be a one parameter family of deformations from $\mathcal{G}$ to $\mathscr{F}$. The $n$th graded part of $\mathrm{App}_{1} \mathscr{F}$ is

$$
\left\{a_{n} t^{n}+a_{n+1} t^{n+1} \mid a_{n} \in F^{n} \mathscr{F}, a_{n+1} \in F^{n+1} \mathscr{F}\right\} .
$$

The multiplication in $\operatorname{App}_{1} \mathscr{F}$ is given by

$$
\begin{aligned}
f_{t}\left(a_{i} t^{i}+a_{i+1} t^{i+1}, b_{j} t^{j}+b_{j+1} t^{j+1}\right) & \\
& =a_{i} b_{j} t^{i+j}+\left(a_{i} b_{j+1}+a_{i+1} b_{j}\right) t^{i+j+1}+t^{i+j+1} F_{1}\left(a_{i}, b_{j}\right) .
\end{aligned}
$$

Denote the elements of $\operatorname{App}_{1} \mathscr{F}$ by $a+b t$. Define an additive section $s$ of the singular extension: $0 \rightarrow \operatorname{sh} \mathcal{G} \rightarrow \operatorname{App}_{1} \mathscr{F} \rightarrow \mathcal{G} \rightarrow 0$ by $s(a)=a+0 \cdot t$. Then

$$
f_{t}(s(a), s(b))-s(a \cdot b)=a \cdot b+t F_{1}(a, b)-a \cdot b=t F_{1}(a, b)
$$

which is the classical infinitesimal of the deformation $f_{t}$. 
We may define

$$
\operatorname{sh}^{n} \mathcal{G}=\bigoplus_{i} F^{i+n} \mathscr{F} / F^{i+n+1} \mathscr{F} \text { and } \operatorname{sh} A_{p p}=\bigoplus_{i} F^{i+1} / F^{i+n+2}
$$

The exact sequence:

$$
0 \rightarrow \operatorname{sh}^{2} \mathcal{G} \rightarrow \operatorname{sh~App} p_{1} \mathscr{F} \rightarrow \mathrm{App}_{1} \mathscr{F} \rightarrow \mathcal{G} \rightarrow 0
$$

is trivial with solution $E_{2}: 0 \rightarrow \operatorname{sh~} A_{p p} \mathscr{F} \rightarrow A_{1} p_{2} \mathscr{F} \rightarrow \mathcal{G} \rightarrow 0$. If the one parameter family of deformations from $\mathcal{G}$ to $\mathscr{F}$ is defined by $g_{t}=\pi+t^{2} F_{2}+$ $t^{3} F_{3}+\ldots$, then $\mathrm{App}_{1} \mathscr{F}$ and $\mathrm{App}_{1} \mathcal{G}$ are isomorphic as filtered rings and therefore $E_{2}^{\prime}: 0 \rightarrow \operatorname{sh} \mathrm{App}_{1} \mathscr{F} \rightarrow \mathrm{App}_{2} \mathcal{G} \rightarrow \mathcal{G} \rightarrow 0$ is also a solution of (1). The equivalence classes of solutions form a principal homogeneous space over $\operatorname{Baer}^{2}\left(\mathcal{G}, \operatorname{sh}^{2} \mathcal{G}\right)$. Thus the difference $E_{2}-E_{2}^{\prime}$ is an element of $\operatorname{Baer}^{2}\left(\mathcal{G}, \operatorname{sh}^{2} \mathcal{G}\right)$ and is equivalent to:

$$
0 \rightarrow \operatorname{sh}^{2} \mathcal{G} \rightarrow \operatorname{App}_{2} \mathscr{F} \times_{\mathrm{App}_{1} \mathscr{F}} \operatorname{App}_{2} \mathcal{G} / \operatorname{diag}\left(\operatorname{sh} \operatorname{App}_{1} \mathscr{F}\right) \rightarrow \mathcal{G} \rightarrow 0 .
$$

This singular extension corresponds to $F_{2}$. In general, if the deformation from $\mathcal{G}$ to $\mathcal{F}$ is defined by $\pi+t^{n} F_{n} t^{n+1} F_{n+1}+\ldots$ for $n>1$, then the singular extension

$$
0 \rightarrow \operatorname{sh}^{n} \mathcal{G} \rightarrow \operatorname{App}_{n} \mathscr{F} \times_{\operatorname{App}_{n-1} \mathscr{F}} \operatorname{App}_{n} \mathcal{G} / \operatorname{diag}\left(\operatorname{sh~} \operatorname{App}_{n-1} \mathscr{F}\right) \rightarrow \mathcal{G} \rightarrow 0
$$

corresponds to $F_{n}$.

The following theorem is the major result of this paper.

THEOREM 2. If $\mathcal{G}$ is a graded algebra (separated and complete) over a field $\mathscr{K}$ of characteristic zero and $\mathcal{G}$ is rigid in the category of algebras, then $\mathcal{G}$ is rigid in the category of filtered algebras.

Proof. Let $\mathcal{F}$ be a filtered algebra over a field $\mathscr{K}$ of characteristic zero whose (complete) associated graded algebra is $\mathcal{G}$. Let $f_{t}=\pi+t F_{1}+t^{2} F_{2}$ $+\ldots$ define a one parameter family of deformations from $\mathcal{G}$ to $\mathscr{F}$ in the category of filtered algebras. We shall show that $f_{t}$ is trivial, i.e. that there exists a filtration preserving algebra isomorphism $\Phi_{t}$ such that $\Phi_{t} \pi(a, b)=$ $f_{t}\left(\Phi_{t} a, \Phi_{t} b\right)$. Since $\mathcal{G}$ is an algebra over a field of characteristic zero and $\mathcal{G}$ is rigid in the category of algebras, $F_{1}$, the infinitesimal of the deformation, must be a coboundary (Gerstenhaber [2], [5]). By Theorem 1, the singular exact sequence:

$$
0 \rightarrow \operatorname{sh} \mathcal{G} \rightarrow \operatorname{App}_{1} \mathscr{F} \stackrel{p}{\rightarrow} \mathcal{G} \rightarrow 0
$$

corresponds to $F_{1}$. Since $F_{1}$ is a coboundary there is a splitting map $s$ : $\mathcal{G} \rightarrow \operatorname{App}_{1} \mathscr{F}$ which does not necessarily preserve filtration. We have $s\left(a_{i}\right)=l_{i}$ $+b_{i}+h_{i}$ where $a_{i} \in \mathcal{G}_{i}, l_{i}=\bigoplus_{k<i} b_{k}, h_{i}=\bigoplus_{k>i} b_{k}, b_{i} \in F^{i} \mathscr{F}$. Since $p$ is gradation-preserving and linear, $l_{i}$ and $h_{i}$ are in the kernel of $p$. If we define $s_{0}: \mathcal{G} \rightarrow \operatorname{App}_{1} \mathscr{F}$ by $s_{0}\left(a_{i}\right)=b_{i}$, then $s_{0}$ preserves filtration and is additive. 
Consider

$$
\begin{aligned}
s\left(a_{i}\right) \cdot s\left(a_{j}\right) & =\left(l_{i}+b_{i}+h_{i}\right) \cdot\left(l_{j}+b_{j}+h_{j}\right) \\
& =l_{i} b_{j}+b_{i} l_{j}+b_{i} b_{j}+b_{i} h_{j}+h_{i} b_{j}
\end{aligned}
$$

with the remaining terms equal to zero since (2) is a singular extension. We have $s\left(a_{i} \cdot a_{j}\right)=s\left(a_{i}\right) \cdot s\left(a_{j}\right)$ and thus $s_{0}\left(a_{i} a_{j}\right)=(i+j)$ th filtered part of $s\left(a_{i}\right) \cdot$ $s\left(a_{j}\right)=b_{i} b_{j}$ by (3). Therefore $s_{0}\left(a_{i} \cdot a_{j}\right)=s_{0}\left(a_{i}\right) \cdot s_{0}\left(a_{j}\right)$. Also $p s_{0}\left(a_{i}\right)=i$ th graded parts of $p s\left(a_{i}\right)$ which is $a_{i}$. Thus sequence (2) splits in the category of filtered algebras and $F_{1}=-\delta \phi_{1}$ for some $\phi_{1} \in C^{1}(\mathcal{G}, \mathcal{G})$ where $\phi_{1}$ preserves filtration. Set $\Phi_{t}(a)=a+t \phi_{1}(a)$. Then

$$
f_{t}\left(\Phi_{t} a, \Phi_{t} b\right)=\Phi_{t}\left(\pi(a, b)+t^{2} F_{2}(a, b)+t^{3} F_{3}(a, b)+\ldots\right) .
$$

By the remarks following Theorem $1, F_{2}$ corresponds to an element of $\operatorname{Baer}^{2}\left(\mathcal{G}, \operatorname{sh}^{2} \mathcal{G}\right)$. The same argument as above shows that this sequence splits in the category of filtered algebras and therefore $\pi+t^{2} F_{2}+t^{3} F_{3}+\ldots$ is equivalent to $\pi+t^{3} F_{3}+\ldots$ in the category of filtered algebras. Induction and the observation that $F_{n}$ corresponds to an element of $\operatorname{Baer}^{2}\left(\mathcal{G}, \operatorname{sh}^{n} \mathcal{G}\right)$ when the deformation is $\pi+t^{n} F_{n}+t^{n+1} F_{n+1}+\ldots$ yields the desired result. Thus we have shown that if a graded algebra is rigid in its operational structure (i.e. rigid as an algebra) then it must also be rigid in its filtration structure (i.e., rigid as a filtered algebra).

\section{REFERENCES}

1. J. P. Coffee, Filtered and associated graded rings, Bull. Amer. Math. Soc. 78 (1972), 584-587.

2. M. Gerstenhaber, On the deformation of rings and algebras, Ann. of Math. 79 (1964), 59-103.

3. On the deformation of rings and algebras. II, Ann. of Math. 84 (1966), 1-19.

4. __ On the deformation of rings and algebras. III, Ann. of Math. 88 (1968), 1-34.

5. $\_$On the deformation of rings and algebras. IV, Ann. of Math. 99 (1974), 257-276.

6. G. Hochschild, On the cohomology groups of an associative algebra, Ann. of Math. 46 (1945), 58-67.

7. U. Shukla, Cohomologie des algèbres associatives, Ann. Sci. École Norm. Sup. Pisa 78 (1961), 163-209.

Department of Mathematics, United States Naval Academy, Annapolis, Maryland 21402 